\title{
Estudio de fallas incipientes en rodamientos usando la técnica de la envolvente y cepstrum
}

\author{
Study on incipient fault bearing detection based on enveloping \\ and cepstrum techniques
}

\author{
Jabid E. Quiroga ${ }^{1} \quad$ Gersón Trujillo $^{1} \quad$ Sergio Quintero $^{1}$ \\ Recibido 1 de marzo de 2011, aceptado 11 de julio de 2012 \\ Received: March 1, 2011 Accepted: July 11, 2012
}

\begin{abstract}
RESUMEN
Este artículo presenta un estudio comparativo del desempeño de los métodos de detección de fallas incipientes en rodamientos FFT, envolvente y cepstrum usando señales de vibración. Diferentes condiciones de fallas son estudiadas, desde incipientes hasta severas a través de un banco de prueba construido para ese propósito. Adicionalmente se utilizan registros de vibraciones obtenidos en el website (CWRU), de Case Western Reserve University, para evaluar el desempeño de las técnicas. Las técnicas de FFT, envolvente y cepstrum implementadas en ambiente Matlab ${ }^{\circledR}$ son aplicadas a las señales de vibración en cada caso de estudio. Envolvente y cepstrum muestran un desempeño satisfactorio en detección de fallas incipientes, ubicándolas como técnicas apropiadas para ser parte de un sistema de monitoreo en máquina rotativa.
\end{abstract}

Palabras clave: Detección de fallas, monitoreo de rodamientos, análisis de vibración, envolvente, cepstrum.

\section{ABSTRACT}

This paper presents a comparative study to determine the performance of FFT, Enveloping and cepstrum incipient bearing fault detection techniques using vibration signal. Different fault scenarios are executed in an experimental test bench from incipient to severe conditions in order to evaluate each technique. Additionally, vibration information given by Case Western Reserve University (CWRU) website is also used to evaluate both techniques. FFT, Enveloping and cepstrum techniques are implemented in Matlab® environment to detect each fault condition. Enveloping and cepstrum provide satisfactory results in fault detection. Therefore, they can be suitable candidates to be used in a rotative machine condition monitoring system.

Keywords: Fault detection, bearings monitoring, vibration analysis, enveloping, cepstrum.

\section{INTRODUCCIÓN}

La falla en rodamientos es uno de los problemas comunes en maquinaria rotativa. Aproximadamente, el 50\% de las fallas en el motor eléctrico, el actuador rotativo más popular, corresponden a fallas en rodamientos [1]. Dentro de las fallas en rodamientos, las fallas asociadas a las pistas representan alrededor del $40 \%$ de las fallas totales en rodamientos [2].
Estas fallas pueden ocasionar importantes pérdidas económicas asociadas a interrupciones no planeadas de la producción, y de un mayor tiempo por cuenta de lo repentino del evento.

Los métodos tradicionales de mantenimiento aplicados a rodamientos se basan en su reemplazo programado a intervalos de tiempo de operación fijos. La periodicidad de este tiempo de operación confiable del rodamiento

1 Escuela de Ingeniería Mecánica. Universidad Industrial de Santander. Cra. 7 Calle 9. Bucaramanga, Colombia. E-mail: jabib@uis.edu.co; gertruna@gmail.com; squintero@gmail.com 
se establece con la información estadística de las fallas. Estos métodos, aunque bastante populares y aún en uso, no son totalmente efectivos debido a que se basan enteramente en una expectativa de tiempo de servicio dado del rodamiento. Sin embargo, muchas fallas resultan por factores impredecibles como un montaje inapropiado, ambiente agresivo, fallas en lubricación, etc. [3]. Por otro lado, el enfoque basado en el mantenimiento programado puede resultar inconveniente cuando el rodamiento es reemplazado prematuramente y constituir un desperdicio de vida de operación del rodamiento.

El esquema de mantenimiento basado en el monitoreo de la condición, $C B M$ de sus siglas en inglés, determina la condición actual de los sistemas basándose en la información que provee este en alguna de sus formas como vibración, sonido, temperatura, entre otras. Dentro de las técnicas más utilizadas para evaluar la condición de los rodamientos en máquinas rotativas se encuentra el análisis de vibraciones [4]. En términos generales, la información transportada por las vibraciones en el dominio de la frecuencia permite determinar posibles condiciones anormales en los rodamientos del equipo rotativo. Adicionalmente, la detección temprana de fallas en rodamientos usando las señales de vibración exige el uso de técnicas apropiadas del campo del procesamiento de señales como la Envolvente y cepstrum [5].

Teniendo como base lo anterior, los autores proponen un estudio comparativo de las técnicas mencionadas arriba para el monitoreo de la condición de los rodamientos en equipo rotativo en distintos escenarios de falla. Las fallas estudiadas cubren fallas en pista externa y pista interna en rodamientos cónicos y de bolas.

La posibilidad de una detección temprana de la condición anormal en los rodamientos es un aspecto importante en el monitoreo de equipo rotativo, por cuanto un diagnóstico en las primeras etapas de la falla puede evitar una mayor afectación del equipo y mejorar la confiabilidad, disponibilidad y mantenibilidad del mismo.

\section{MARCO TEÓRICO}

En condiciones normales de operación, es decir, buena alineación y sin sobrecarga, sobrevienen las fallas debido a fatiga del rodamiento. Estas fallas en sus primeras etapas se localizan por debajo de la superficie de las pistas y los elementos rodantes. En una etapa posterior la afectación se propaga hacia el exterior produciendo grietas y picaduras que a su vez desencadenan una elevación en la vibración y los niveles sonoros de operación del rodamiento [6].

Una vez la falla avanza hacia la superficie el área afectada se expande rápidamente contaminando el lubricante y causando sobrecargas localizadas sobre las superficies de las pistas. Eventualmente, la falla evoluciona produciendo una superficie rugosa en las pistas y los elementos rodantes [7].

Aunque el modo normal de falla en rodamientos es por fatiga, existen otras condiciones externas que pueden afectar la vida útil del rodamiento como la contaminación, lubricación e instalación inapropiadas [8].

\section{Monitoreo de rodamientos usando análisis de vibración}

El monitoreo de rodamientos se basa principalmente en la observación del contenido de información en amplitud y frecuencias de la señal de vibración. Incrementos en magnitud o excitación de algunos armónicos son síntomas de la presencia de condiciones anormales en los rodamientos La producción de la sintomatología en señales de vibración, asociada a fallas en pista externa e interna en rodamientos, se describe como la excitación del sistema formado por el rodamiento, su carcasa y el eje de la máquina todos actuando como una campana en resonancia. Cuando se presenta un desperfecto en la pista interna o externa del rodamiento, el elemento rodante produce un pequeño impulso de baja energía, provocando que el rodamiento vibre. Este impulso excita la frecuencia natural del conjunto cuyo valor depende de las propiedades de los materiales y la geometría del sistema. Esta vibración es atenuada por el amortiguamiento del sistema, decayendo rápidamente. Durante la operación, con cada revolución, un número específico de elementos rodantes golpean el defecto en la pista, provocando un tren de respuestas, ver Figura 1.

Las frecuencias de falla asociadas al defecto en la pista interna $B P F I$ y pista externa $B P F O$ en $\mathrm{Hz}$ se muestran en (1-2) [5]. Estas frecuencias de falla dependen de la geometría del rodamiento y de la velocidad de rotación de este. 


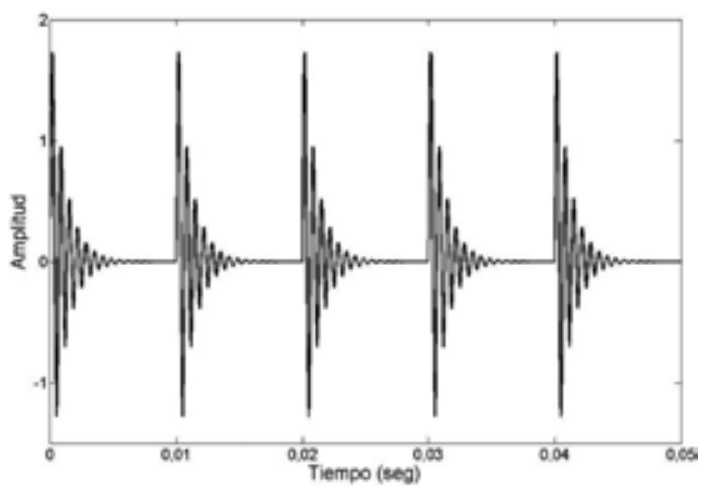

Figura 1. Representación de la señal de falla adquirida en el rodamiento.

$$
\begin{aligned}
& B P F I=\frac{R P M}{60}\left(\frac{N_{b}}{2}\right)\left(1+\frac{B_{d} \cos \phi}{P_{d}}\right) \\
& B P F I=\frac{R P M}{60}\left(\frac{N_{b}}{2}\right)\left(1-\frac{B_{d} \cos \phi}{P_{d}}\right)
\end{aligned}
$$

donde $R P M$ es la velocidad del rodamiento en revoluciones por minuto, $B_{d}$ es el diámetro del elemento rodante, $N_{b}$ corresponde al número de elementos rodantes, $P_{d}$ es el diámetro efectivo del rodamiento y $\phi$ es el ángulo de contacto tomado desde la línea central del elemento rodante y el eje del rodamiento.

Las frecuencias de falla, determinadas usando (1-2), presentan ligeras variaciones con respecto a los valores experimentales; debido principalmente al desplazamiento de las superficies (las pistas) o pérdida de contacto de la pista con el elemento rodante.

Dentro de las herramientas disponibles para determinar la información que contiene una señal de vibración, la más utilizada corresponde a la Transformada Rápida de Fourier (FFT), la cual es utilizada en este estudio. Aunque efectiva en la determinación de armónicos y amplitudes, la FFT no permite detectar fallas en pista exterior o interior de rodamientos cuando éstas están en su etapa inicial, debido a que la frecuencia excitada por la condición de falla es de baja amplitud y alta frecuencia con lo cual se enmascara como ruido de alta frecuencia.

\section{Técnica de la envolvente}

Debido a la presencia de defectos localizados, se producen cambios abruptos en los esfuerzos de contacto en las interfaces, lo cual resulta en la generación de pulsos de muy corta duración que excitan las frecuencias naturales de las pistas y soporte del rodamiento. La técnica de la envolvente busca principalmente detectar zonas resonantes excitadas o moduladas por fuerzas de impacto periódicas, cuya frecuencia de repetición es un indicador del lugar donde se encuentra el defecto y su amplitud una medida del defecto y progresión de la falla.

El tren de pulsos, que ocasiona el impacto del elemento rodante sobre la pista defectuosa, puede verse como una señal portadora (dada a altas frecuencias debido a la resonancia de la carcasa del rodamiento) modulada en amplitud por la envolvente. Esta envolvente corresponde a la atenuación de la respuesta al impulso producto del amortiguamiento del conjunto [9]. La frecuencia de interés en la detección de los defectos de rodamientos es la frecuencia de modulación, la cual corresponde a los valores de $B P F O$ y $B P F I$.

La técnica de la envolvente permite extraer las frecuencias de interés a partir de la definición de la señal modulada. Este proceso implica el uso de una secuencia de operaciones con la señal de vibración que inicia con la eliminación de componentes de baja frecuencia asociadas a otras condiciones del equipo rotativo, como desbalanceo o desalineamiento. Posteriormente, se demodula la señal a través de la Transformada de Hilbert, encargada de rectificar la señal y determinar la señal envolvente con una frecuencia que corresponde a los valores de $B P F O$ y BPFI. Como última etapa se eliminan las altas frecuencias y se obtiene del espectro de la señal resultante [9].

\section{Cepstrum}

El procesamiento de señales usando cepstrum es una técnica no lineal con una amplia variedad de aplicaciones en áreas tales como; el procesamiento de voz, imágenes y monitoreo de equipos. El análisis empleando cepstrum es usado para determinar periodicidades en el análisis espectral de una señal y para separar el efecto de funciones de transferencia variables en el tiempo en una convolución de señales [10]. Los picos en el cepstrum, "rahmonics", son un indicador de la presencia de armónicos en el 
espectro y su ubicación en el eje "Quefrency" del cepstrum corresponde al periodo de la señal en el dominio del tiempo. El cepstrum real de una señal $C(t)$ es comúnmente llamado cepstrum y se determina usando (3) [11].

$$
C(q)=\operatorname{real}\left(F T^{-1}(\log [F T(C(t))])\right)
$$

donde $F T^{-1}$ y $F T$ son la Transformada Inversa de Fourier y la Transformada de Fourier de la señal de entrada $C(t)$ respectivamente. Debido a la operación logarítmica aplicada a la magnitud del espectro de la señal, componentes pequeños asociados al fenómeno de fuga de frecuencias "Leakage" son amplificados y afectan el cepstrum resultante. Por esta razón en [11] se propone una versión modificada denominada Cepstrum Lineal (LCEPS), en el cual la operación logarítmica no es aplicada al cepstrum real resultando (4).

$$
\operatorname{LCEPS}(q)=\operatorname{real}\left(F T^{-1}(F T(C(t)))\right)
$$

Este nuevo enfoque aplicado a la señal de vibración, en la detección de la $B P F O$ y la $B P F I$, ofrece resultados prometedores por cuanto elimina componentes no significativos de la señal; que pueden dificultar el proceso de identificación de los rasgos buscados en esta. En este trabajo se utiliza $L C E P S$ para analizar la señal de vibración.

\section{METODOLOGÍA}

El proceso de implementación de la técnica de la envolvente, para la detección de fallas en rodamientos, se inicia con una simulación en Matlab ${ }^{\circledR}$ del comportamiento dinámico del rodamiento cuando este presenta un defecto en su pista externa, para lo cual se simula la señal armónica a la frecuencia natural del conjunto de rodamiento con soporte, ver (5). La anterior señal decae como resultado del amortiguamiento asociado al sistema y oscila con un periodo de 0,05 segundos. Posteriormente se aplica la técnica a datos que se obtienen del website [12]. Los registros de vibraciones almacenados en este website corresponden a la señal de vibración de un banco que presenta diferentes severidades de falla en la pista externa e interna de rodamientos de bolas. Por último, se valida el algoritmo en un banco experimental implementado para este propósito.
La señal simulada con (5) en dominio tiempo se muestra en la Figura 1 y su espectro usando la $F F T$ se muestra en la Figura 2, en donde se puede observar que la frecuencia asociada a la $B P F O$, es decir, $20 \mathrm{~Hz}$, no se define claramente.

$$
y=2 e^{-400 t} \sin (2 \pi * 100 t)
$$

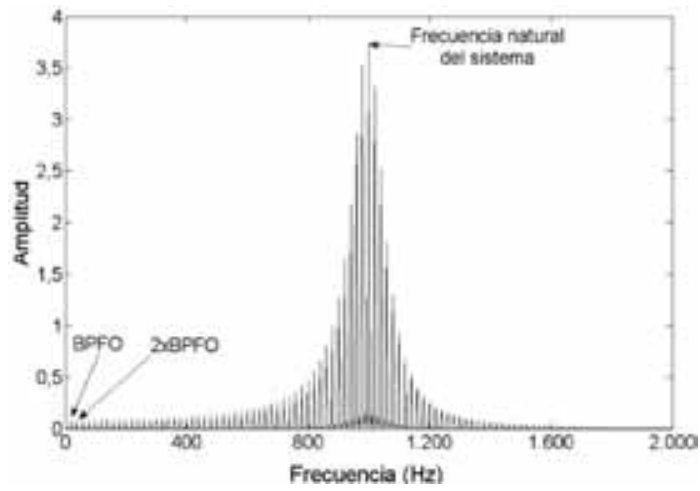

Figura 2. Espectro de la señal simulada.

El proceso de demodulación, base de la técnica de la envolvente, consiste en obtener a partir de la vibración original una nueva señal que contenga únicamente la señal modulada. Esta nueva señal también se conoce como envolvente de la señal original. El espectro de la señal demodulada se denomina espectro de demodulación y para el caso de la señal simulada en (5) se muestra en la Figura 3. El espectro de demodulación obtenido contiene picos armónicos, amplificados en el caso de la frecuencia de $B P F O$ de 260 veces respecto al valor obtenido con la $F F T$ de la señal de vibración original.

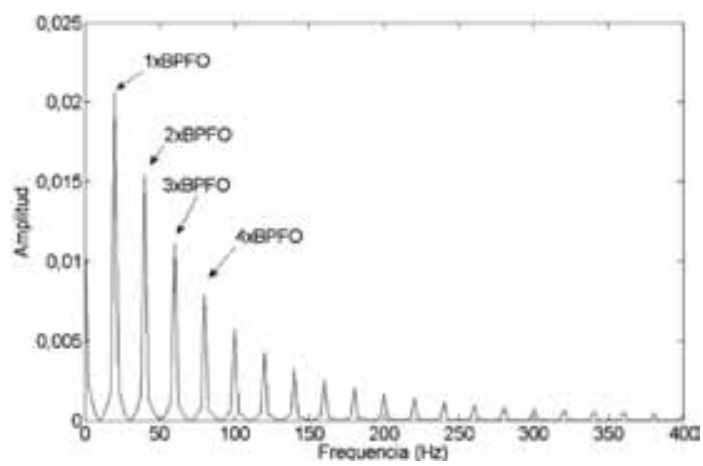

Figura 3. Espectro de la envolvente para la señal simulada. 
La técnica envolvente ahora es aplicada a los registros disponibles en [12] que corresponden a señales de vibraciones para un rodamiento de bolas 6205-2RS $J E M S K F$ instalado en un banco de pruebas. El banco posee un motor de $2 \mathrm{Hp}$, un transductor/encoder de torque, un dinamómetro y controles electrónicos. El acelerómetro es ubicado en la posición centrada del apoyo. El eje gira a $1772 R P M$ y los grados de severidad en la pista externa e interna son: Un primer grado para una falla de profundidad de 0,007 ', y un segundo grado de falla de 0,028 ".

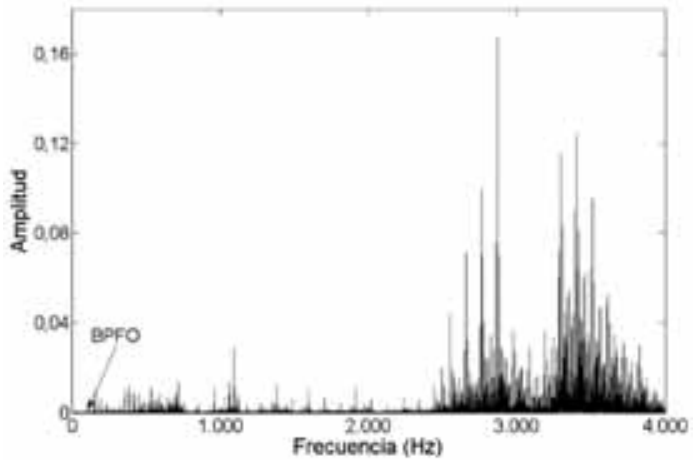

Figura 4. Espectro de la señal de vibración para defecto incipiente en pista externa.

La Figura 5 enseña el espectro del primer escenario usando la técnica de la envolvente:

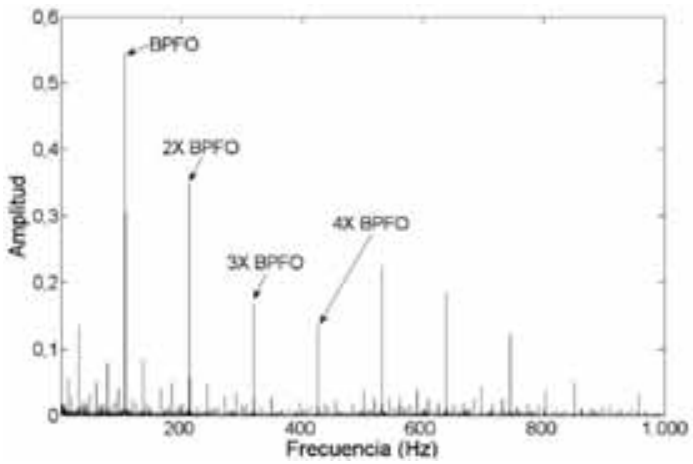

Figura 5. Espectro de la señal envolvente para pista externa con falla incipiente.

En la Figura 6 se muestra el espectro de la señal de vibración para el segundo escenario de falla $(0,028 ")$ usando la FFT:

Nuevamente, se aplica la técnica de la envolvente a la señal y se obtiene el espectro de la Figura 7.

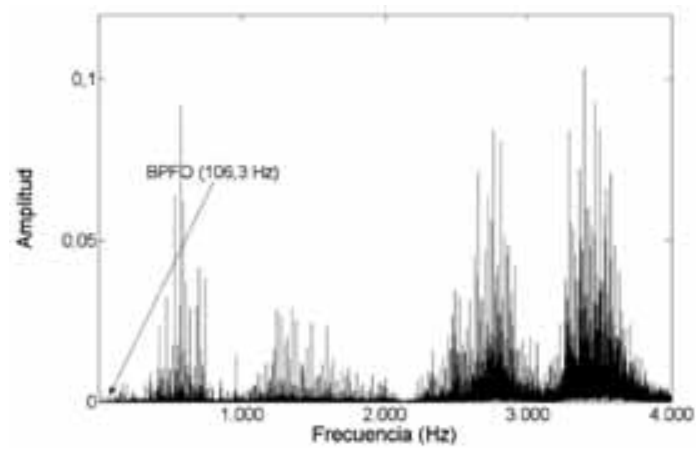

Figura 6. Espectro de la señal de vibración para falla avanzada en pista exterior.

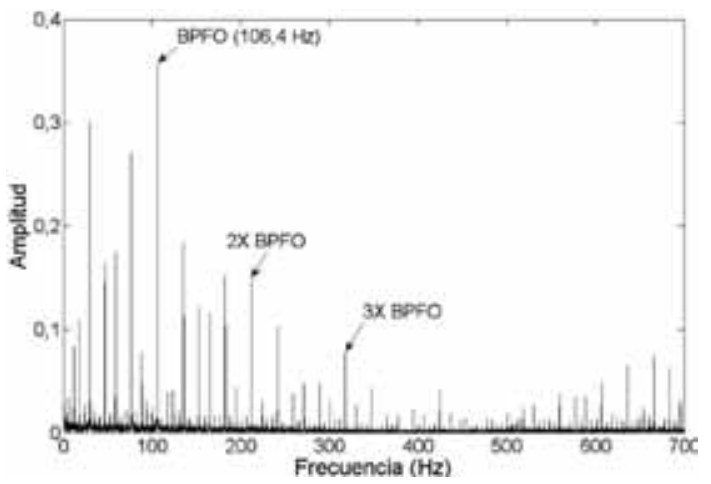

Figura 7. Espectro de la señal envolvente para pista externa con falla avanzada.

Lo observado en las figuras permite concluir que con el uso de la FFT para las señales, las frecuencias $B P F O$ son opacadas por la gran amplitud de los armónicos de alta frecuencia correspondientes en su mayoría a la frecuencia natural del sistema y al ruido. Por otra parte, al aplicar la técnica de la envolvente y observar y comparar los espectros con los obtenidos mediante la FFT, se concluye que la técnica de la envolvente permite identificar claramente y amplifica las frecuencias $B P F O$ y sus armónicos.

A continuación se muestran los resultados obtenidos para los dos escenarios de falla, esta vez en la pista externa. La Figura 8 muestra el espectro obtenido con la FFT para el primer escenario ( 0,007 ").

Por su parte la Figura 9 enseña el espectro de la señal del mismo escenario anterior usando la técnica de la envolvente: 


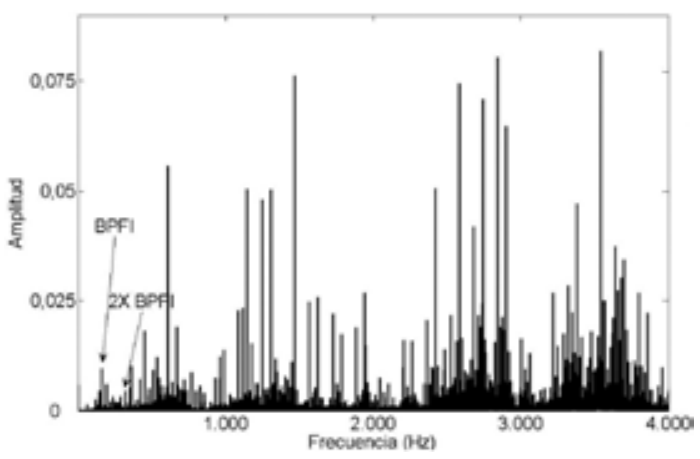

Figura 8. Espectro de la señal de vibración para defecto incipiente pista interna.

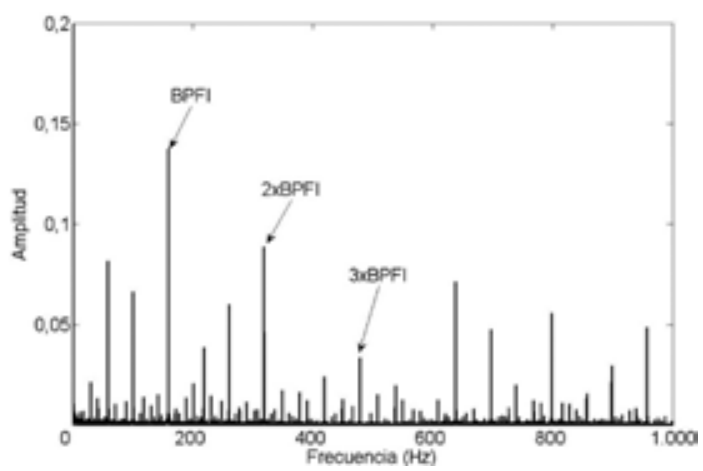

Figura 9. Espectro de la señal envolvente para defecto incipiente pista interna

Ahora, para el segundo grado de severidad de la falla en pista interna se obtiene el espectro a través de la FFT (ver Figura 10).

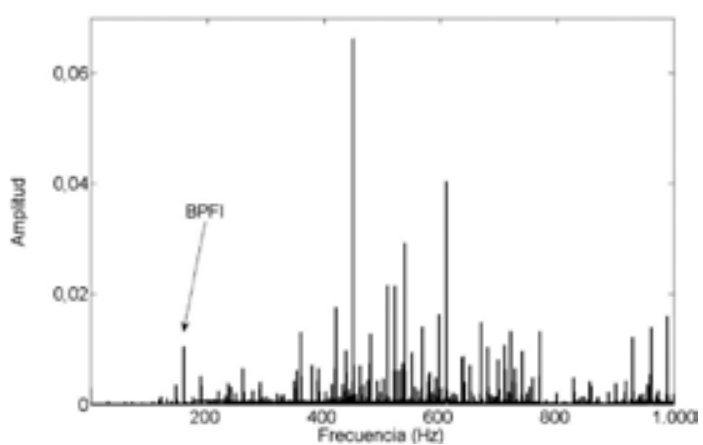

Figura 10.Espectro de la señal de vibración para defecto severo pista interna.

Nuevamente, se emplea la técnica de la envolvente para obtener el espectro de la misma señal (ver Figura 11) en el cual se observan con mayor claridad las frecuencias asociadas a BPFI.

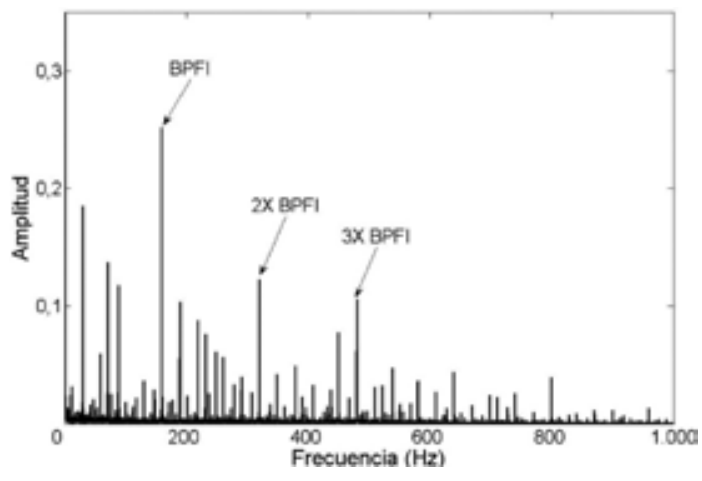

Figura 11.Espectro de la señal envolvente para defecto severo pista interna.

El experimento para la falla en la pista interna del rodamiento arroja que las frecuencias BPFI son apenas visibles en el espectro obtenido con la FFT, en donde predominan los componentes de alta frecuencia. Sin embargo, al tratar las mismas señales con la técnica de la envolvente, al igual que sucedió en el caso de la falla en pista externa, los componentes de frecuencia asociados a la BPFI tienen mayor amplitud con respecto a los demás componentes del espectro y esto permite identificarlos claramente.

\section{Validación experimental de la envolvente}

En la presente sección se describe la validación experimental de la técnica utilizando un banco de ensayos para rodamientos cónicos del Laboratorio de Vibraciones de la Universidad Industrial de Santander (UIS). El banco posee tres apoyos tipo rodamientos y un motor de $1 / 8 h p$ y $1800 R P M$. En el extremo libre del eje se ubica el rodamiento en prueba (rodamiento cónico SKF 30203). Se emplean tres apoyos con el fin de mantener la alineación cada vez que se manipula el rodamiento en prueba (ver Figura 12).

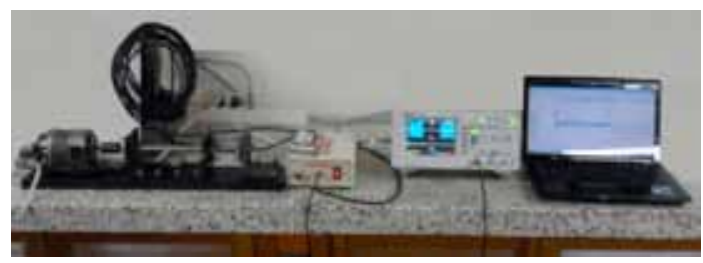

Figura 12. Banco de prueba utilizado.

La señal de vibración se captura a través de un sistema de adquisición de datos de 24 bits marca National Instruments a una frecuencia de muestreo 
de $5 \mathrm{KHz}$. El acelerómetro modelo 325C34 de $P C B$ Piezotronics con un ancho de banda de $0,5-10 \mathrm{KHz}$ $\mathrm{y}$ un rango de medición de $\pm 50 \mathrm{~g}$. Adicionalmente, se utiliza un mazo de impulso, $P C B$ Piezotronics modelo $086 \mathrm{C} 01$, con una resolución de (+/- $440 \mathrm{~N}$ pk) y una frecuencia resonante: $>15 \mathrm{KHz}$.

Para establecer la ubicación óptima del acelerómetro, es decir, aquella en la cual la energía de vibración se manifieste con mayor amplitud, se determinan los modos de vibración del banco modelado a escala real, utilizando ANSYS Workbench. Una vez hecho el análisis, se concluye que la dirección axial refleja la mayor amplitud de vibración. Adicionalmente, se determinan experimentalmente las frecuencias naturales del sistema con el propósito de diseñar los filtros necesarios para implementar la técnica de la envolvente. Las frecuencias naturales del banco de prueba determinadas son 738, 847 y $1145 \mathrm{~Hz}$ respectivamente.

La implementación de la técnica de la envolvente implica un tratamiento de la señal de vibración. En la primera etapa se eliminan componentes de baja frecuencia a través de un filtro tipo Butterworth pasa-altas de orden 10 y con frecuencia de corte igual a los $1000 \mathrm{~Hz}$ (esta frecuencia de corte debe estar por debajo de la máxima frecuencia natural que presente el sistema aproximadamente $1145 \mathrm{~Hz}$ ). Posteriormente, la señal filtrada se demodula usando la Transformada de Hilbert, y por último, se eliminan frecuencias no relevantes para el monitoreo de rodamientos empleando un filtro tipo Butterworth pasa-bajas de orden $10 \mathrm{y}$ de frecuencia de corte igual a los $600 \mathrm{~Hz}$ para visualizar los primeros tres armónicos de la $B P F O, 182,37 \mathrm{~Hz}$.

Los escenarios estudiados en esta etapa experimental corresponden a rodamiento sin falla, rodamiento con falla incipiente (ver Figura 13-a), rodamiento con falla intermedia (ver Figura 13-b) y por último rodamiento con falla avanzada (ver Figura 13-c).
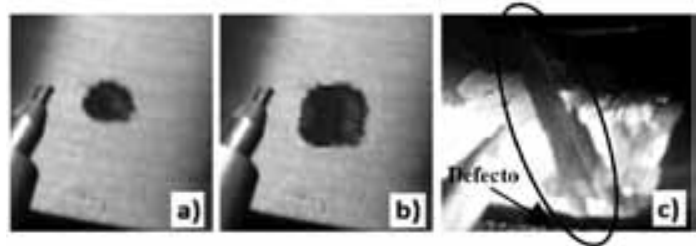

Figura 13. Defectos en la pista externa: a) incipiente, b) intermedia y c) avanzada
Se ubica en la fotografía una punta de grafito de diámetro $0,5 \mathrm{~mm}$ para comparar su tamaño con el tamaño del defecto inducido.

Los defectos incipiente e intermedio fueron realizados mediante el choque eléctrico de un electrodo con la pista externa, provocando el descascaramiento de una parte de la pista externa. El defecto avanzado se obtuvo con un disco de esmeril afectando severamente la pista externa. Estas fallas corresponden a la progresión del descascarado en los rodamientos.

A continuación se mostrarán los espectros correspondientes al análisis de vibraciones para cada escenario de falla estudiado. En las Figura 14 y 15 se muestran los espectros para el rodamiento sin falla. En la Figura 14 se observan picos correspondientes a la frecuencia natural del sistema y a penas se puede distinguir la BPFO. En la Figura 15 se muestra un gran número de armónicos excitados por la rotación del rodamiento.

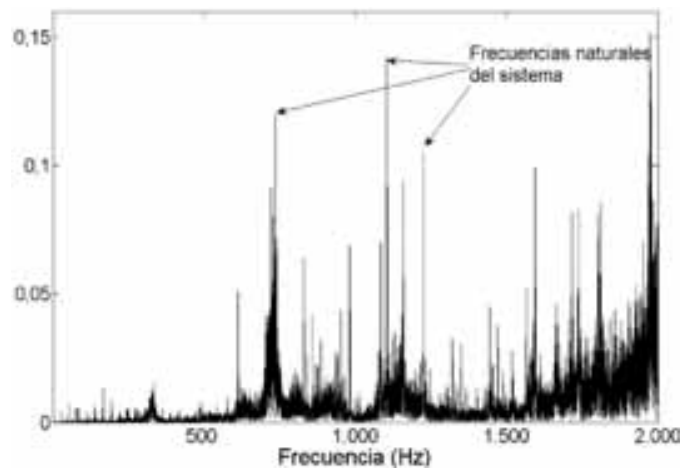

Figura 14. Espectro de la vibración para rodamiento sin falla.

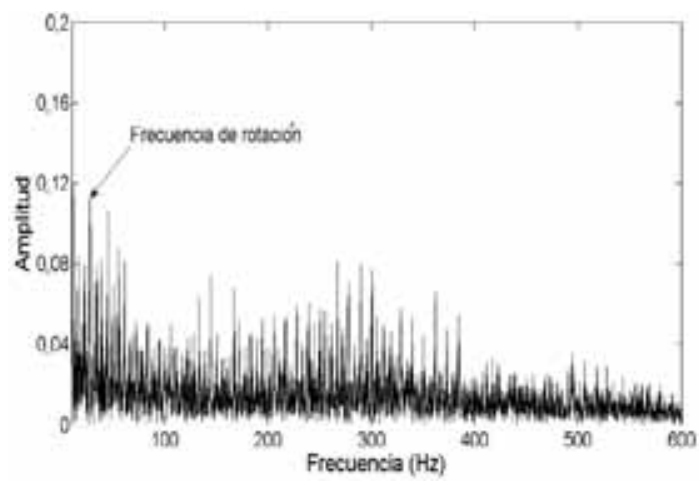

Figura 15. Espectro de la envolvente para rodamiento sin falla. 
En las Figuras 16,18 y 20 se muestran los espectros para los rodamientos con fallas en pista externa incipiente, intermedia y avanzada, respectivamente. En la Figura 16, la $B P F O$ posee una amplitud que la ubica prácticamente imperceptible en el espectro. En la Figura 18 aparece más visible la frecuencia de falla pero con una magnitud muy pequeña, dificultando el proceso de identificación de la falla. En la Figura 20 se observa que el espectro permite la identificación de la condición de falla, mostrando la $B P F O$ claramente.

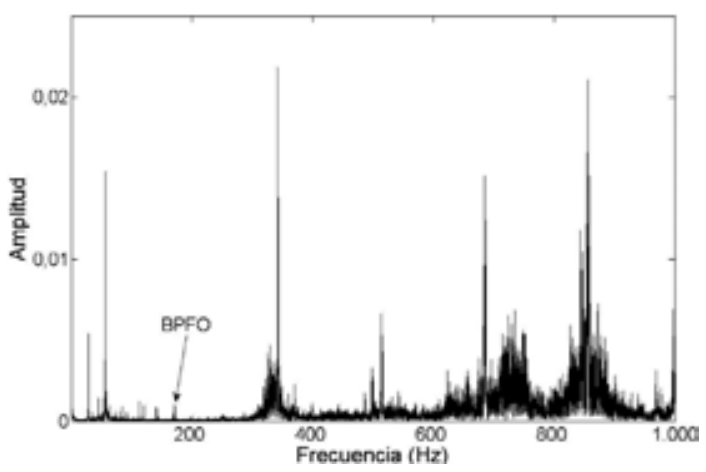

Figura 16. Espectro de la vibración para rodamiento con defecto en la pista externa incipiente.

En las Figuras 17,19 y 21 se muestran los espectros de las envolventes para los rodamientos con fallas incipiente, intermedia y avanzada respectivamente. En los espectros mostrados se pueden diferenciar claramente la $B P F O$ y sus armónicos. Sin embargo, en la condición de falla avanzada se observa que las magnitudes de las $B P F O$ y sus armónicos son menores que las del espectro obtenido sin aplicar la técnica de la envolvente.

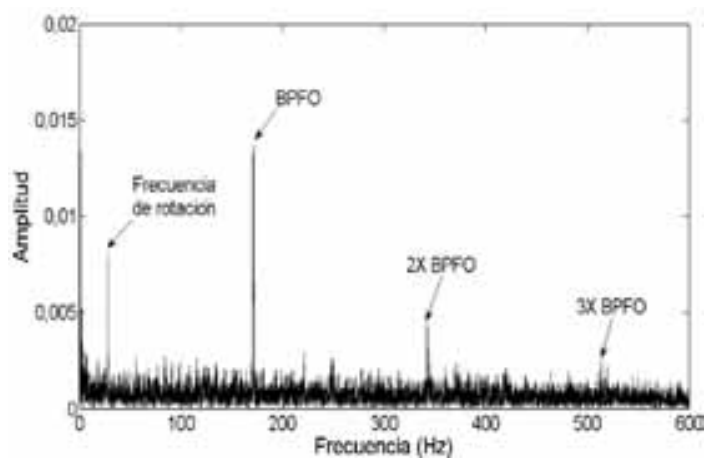

Figura 17. Espectro de la envolvente para rodamiento con defecto en la pista externa incipiente.

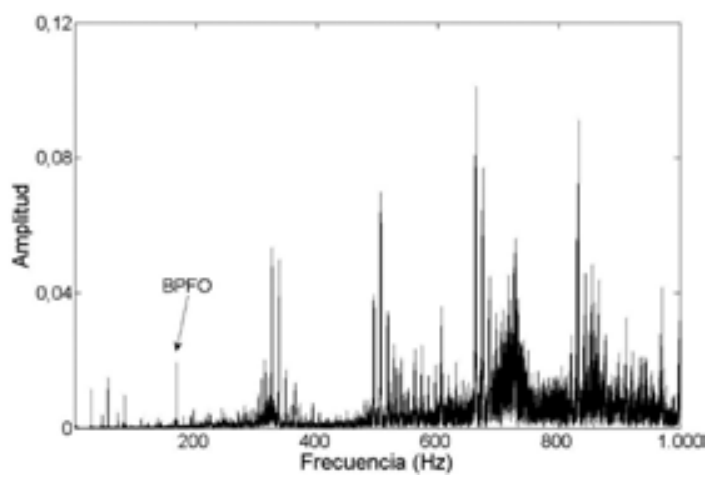

Figura 18. Espectro de la vibración para rodamiento con defecto intermedia en la pista externa.

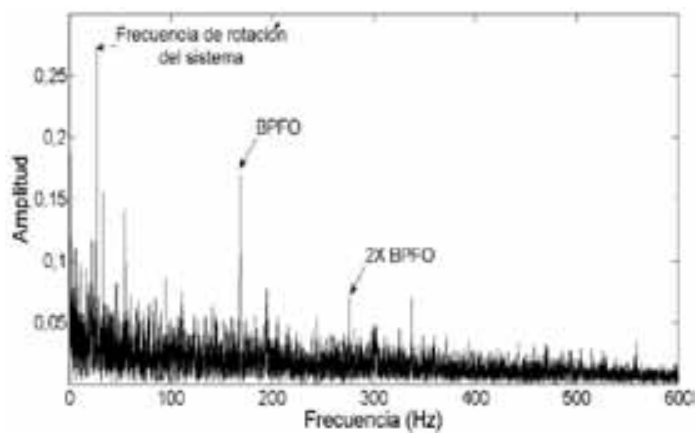

Figura 19. Espectro de la envolvente para rodamiento con defecto intermedia en la pista externa.

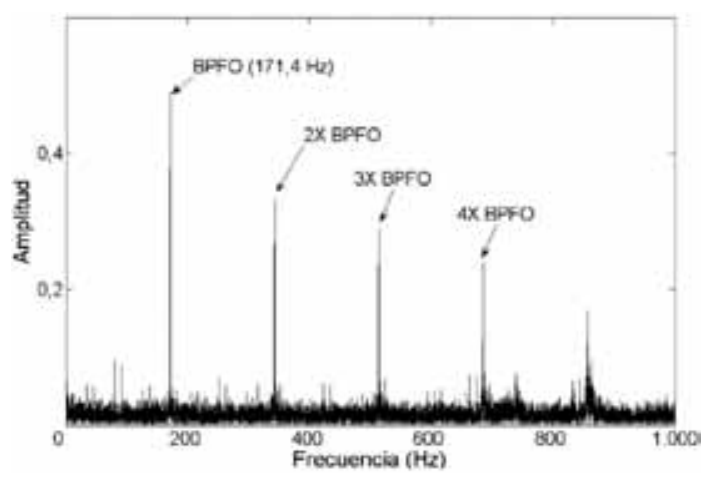

Figura 20. Espectro de la vibración para rodamiento con defecto avanzada en la pista externa.

\section{Validación experimental de la cepstrum}

El proceso de implementación de esta técnica empleando los datos experimentales obtenidos en el banco de pruebas requiere del filtrado del registro de vibración. El filtrado se realiza con un filtro pasabandas tipo Butterworth con un ancho de banda de $40 \mathrm{~Hz}$ alrededor de la $B P F O$ teórica con lo cual se eliminan las componentes en frecuencia 
no relevantes para el monitoreo de la condición del rodamiento en prueba.

En la Figura 22 se muestra el cepstrum para cada una de las señales de vibración correspondientes a los casos implementados, es decir, rodamiento sin falla, con falla incipiente, con falla intermedia y por último rodamiento de falla avanzada.

Como se puede observar en la Figura 22, la magnitud de los "rahmonics" presentes corresponde a la frecuencia aproximada de $B P F O(172,41 \mathrm{~Hz})$ y sus armónicos. Adicionalmente, se nota que en la medida que se aumenta el nivel de severidad de la falla en la pista exterior mayor es la magnitud asociada al "rahmonic" fundamental. Este comportamiento permite candidatizar a este análisis y a la magnitud del "rahmonic" fundamental para el monitoreo de esta condición de falla.

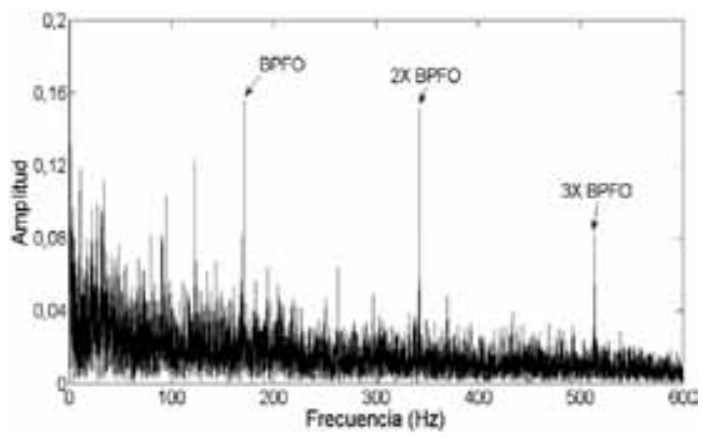

Figura 21. Espectro de la envolvente para rodamiento con defecto en la pista externa avanzada.

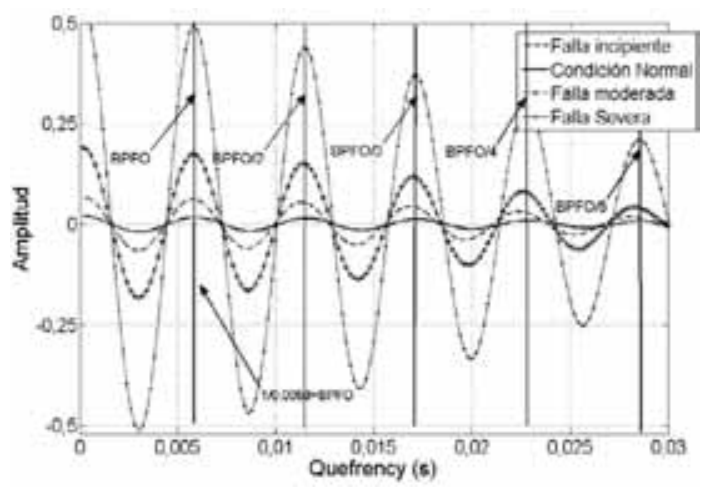

Figura 22. Cepstrum para rodamientos en prueba.

\section{ANÁLISIS DE RESULTADOS}

Los resultados experimentales para el caso de la técnica de la envolvente para fallas en pista exterior e interior mostraron ser consistentes con el escenario de falla estudiado. Es decir, en presencia de falla se activaron las frecuencias $B P F O$ y $B P F I$ y sus respectivos armónicos. Las magnitudes de estas frecuencias de falla son proporcionales a la severidad de las fallas y se obtienen ganancias sustanciales en magnitud hasta de 13 cuando la técnica es implementada. El único caso anómalo correspondió a la falla severa para la pista externa, en la cual, aunque se discrimina perfectamente la $B P F O$ y sus armónicos, el uso de la técnica atenuó la magnitud de la frecuencia de falla, concluyéndose con ello que la técnica de la envolvente tiene un mejor desempeño cuando las fallas son incipientes y moderadas. Así, esta es la limitación del método, a medida que el defecto pierda el carácter de impulsivo, este dejará de estimular las frecuencias naturales del rodamiento y con esto la detección del defecto. En el caso del rodamiento sin falla, en el espectro se observan las frecuencias naturales del sistema y picos en la frecuencia característica de falla en la pista externa e interna, ambas de baja amplitud. Estas señales de vibración se atribuyen a pequeñas imperfecciones en el diseño o defectos de fábrica. En el caso del cepstrum se presentó activación de las frecuencias de falla y hubo consistencia entre la magnitud del armónico fundamental $(B P F O)$ y la severidad de la falla en todos los escenarios de prueba.

\section{CONCLUSIONES}

En este artículo se ha presentado un estudio comparativo entre tres métodos de monitoreo de fallas en pista externa e interna para rodamientos de bolas y cónicos, los cuales se han establecido como técnicas para detección de fallas en rodamientos. Aunque la experimentación se realizó con rodamientos cónicos y de bolas, las conclusiones de este estudio pueden hacerse extensivas a los demás tipos de rodamiento, en cuyo caso los valores de $B P F O$ y $B P F I$ serán los calculados de acuerdo a la configuración geométrica del rodamiento estudiado. De acuerdo a los resultados obtenidos, la técnica de la envolvente y el cepstrum modificado pueden ser implementados satisfactoriamente para el monitoreo de falla de rodamientos, en pista externa e interna, cuando se requiere una detección temprana y más aún para evaluar el grado de severidad de la falla debido a que se encontró que la magnitud de la frecuencia fundamental de BPFI y $B P F O$ se eleva con la severidad de la falla. La $F F T$ mostró ser efectiva para 
detectar fallas severas. Adicionalmente, se encontró que cuando la falla se torna severa la técnica de la envolvente menoscaba el monitoreo por cuanto atenúa la $B P F O$ y sus armónicos. Contrariamente, el cepstrum mostró ser consistente en magnitud con el nivel de severidad de la falla a lo largo de los diferentes niveles de severidad experimentados. Por otro lado, se confirmó el desempeño superior del algoritmo $L C E P S$ con respecto al cepstrum real en la identificación de la $B P F O$ para este tipo de fallas.

\section{REFERENCIAS}

[1] P.F. Albrecht, J.C. Appiarius, R.M. McCoy, E.L. Owen and D.K. Sharma. "Assessment of the reliability of motors in utility applicationsUpdated". IEEE Trans. Energy Conversion. Vol. 1, pp. 39-46. March, 1986.

[2] B. Raison, G. Rostaing, O. Butscher and C. Maroni. "Investigations of algorithms for bearing fault detection in induction drives". 28th Annual Conference of the Industrial Electronics Society. Vol. 2, pp. 1696-1701. November, 2002

[3] T.R. Kurfess, S. Billington and S. Liang. "Advanced Diagnostic and Prognostic Techniques for Rolling Element Bearings". Springer Series in Advanced Manufacturing. Ed. Springer. London, pp. 137-165. 2006.

[4] Z. Reif and M.S. Lai. "Detection of Developing Bearing Failures by Means of Vibration". Rotating Machinery Dynamics. Ed. The American Society of Mechanical Engineers. New York, pp. 231-236. 1989
[5] J. Fernandez, A. Bediaga, I. Gaston and A. Hernandez. "Evaluation Study on Detection Techniques for Bearing Incipient Faults". The International Conference on Computer as a Tool. Vol. 2, pp. 1566-1569. November, 2005.

[6] P. Eschmann, L. Hasbargen and K. Weigand. "Ball and Roller Bearings: Their Theory, Design, and Application”. Ed. K. G. Heyden. London, pp. 147-149. 1958.

[7] J. Riddle. "Ball Bearing Maintenance". Norman. Ed. University of Oklahoma. Oklahoma, pp. 30-32, 1955

[8] R. R. Schoen, T. G. Habetler, F. Kamran, R. G. Bartfield. "Motor bearing damage detection using stator current monitoring". IEEE Transactions on Industry Applications. Vol. 31, Issue 6, pp. 1274-1279, Noviembre 1995.

[9] S. A. McInerny, Y. Dai. "Basic vibration signal processing for bearing fault detection". IEEE Transactions on Education. Vol. 46 (1), pp. 149-156, Febrero 2003.

[10] A. V. Oppenheim, R. W. Schafer. "From frequency to quefrency: a history of the cepstrum". IEEE Signal Processing Magazine. Vol. 21(5), pp. 95- 06, Septiembre 2004.

[11] N. T. Van der Merwe, A . J. Hoffman. "A modified cepstrum analysis applied to vibration Signals". International Conference on Digital Signal Processing. Vol. 2, pp. 873876, Noviembre 2002.

[12] http://www.eecs.case.edu. Fecha de consulta el 10 de diciembre de 2009. 